\title{
Accidental mercury spill in the Andes: forensic neuropsychological evaluation six years later
}

\author{
F. Gonzalez \& A. Saldivar \\ Rancho Los Amigos National Rehabilitation Center, USA
}

\begin{abstract}
On June 2, 2000, 330 pounds of elemental mercury from a local gold mine was spilled over a 45-kilometer stretch of the region's main road. The affected road represents the only route between towns and receives heavy foot traffic. People from the surrounding towns, believing the mercury to be valuable, gathered and carried mercury using any means available. They took mercury into their homes and in many cases handled mercury extensively, even going so far as to try to clean it. Adults and children of all ages were exposed. Many were hospitalized and chelated; some were not. Levels of exposure and symptoms varied, and many victims did not receive treatment or care as local resources were quickly overwhelmed. This paper outlines an approach to a forensic assessment model for a culturally diverse and challenging population. The literature on the neuropsychological effects of mercury on children and adults will be briefly discussed. Recommendations from the World Health Organization for assessment of neurotoxicity and their implementation in the current project will be reviewed. Two pediatric cases are presented from a medical and neuropsychological perspective. Discussions regarding the effects of the spill on the local educational system and community as a whole will be presented. Keywords: neuropsychology, culture, Spanish, mercury exposure, toxic exposure, forensic, neurotoxicity, chelation, penicillamine, child, pediatric.
\end{abstract}

\section{Introduction}

Mercury (Hg) was known to ancient Hindus and Chinese before 2000 B.C. The Greeks used it in ointments, the Romans in cosmetics, and it has been found in 3500 year old Egyptian tombs. It is often a by-product of gold mining and frequently used in thermometers, barometers and pressure sensing devices, 
batteries, lamps, dental amalgam, pharmaceuticals, religious practices, etc. Mercury easily forms alloys with other metals and is frequently used to extract gold from its ores. One of the world's largest goldmines is located in the Andes in a town called Yanacocha, located in the northern part of Peru. In June 2000, while transporting mercury that was a by-product of the gold mining process, 330 pounds of elemental mercury was spilled over a 45 -kilometer stretch of the region's main road. The affected road is the only route between towns and is travelled heavily by foot by the local inhabitants, most of whom cannot afford cars. When people in the area became aware that there were glittering silver droplets on the road, they began to gather the mercury thinking it could be valuable. They collected the mercury using bottles, cans, cups, brooms, pockets, even their hands. People took the mercury into their homes and in many cases handled it extensively, with some individuals even trying to clean it. Over 1000 adults and children were exposed. When the local authorities became aware of the spill, attempts were made at cleaning the contaminated areas and treating the exposed individuals. A protracted legal battle ensued that is currently ongoing and numerous medical studies were initiated to assess the effects of the mercury exposure on the affected population. Given the cultural and geographic issues, and limited studies, there were few existing protocols or specific guidelines addressing appropriate neuropsychological evaluation of mercurial exposure. The following paper will discuss the development, application, and results of a forensically oriented neuropsychological assessment of a sample of the exposed individuals.

\section{Deficits commonly found with mercury exposure}

Elemental/metallic mercury $(\mathrm{Hg} 0)$ is a silvery metal with high vapor pressure that can exist either as a liquid or vapor form in ambient conditions. Elemental mercury is absorbed through the lungs and crosses the blood-brain barrier, the placenta, and other tissues prior to oxidation. Oxidation changes the elemental mercury into inorganic mercury, which cannot cross the blood-brain barrier and is therefore trapped by oxidation within the brain [1]. Rohlings and Demakis [2] report that exposure to elemental mercury can result in tremors, psychological problems (e.g., erethism manifested as anxiety, timidity, shyness, and mood lability), and higher order neuropsychological impairments (including deficits in attention, intellectual disturbances, visuospatial abilities, cognitive abstraction, reaction time, visual memory, and decreased cognitive efficiency).

Given the nature and history of research into mercury exposure, it has become an excellent experimental model for studying the developmental neurotoxicity of heavy metals. Previous research as cited by Deitch [3] noted substantial deficits in visual perceptual constructional skill, nonverbal memory and abstract reasoning related to exposure to $\mathrm{Hg}$ vapors in a home by children. Though follow-up testing at one year revealed some improvement, deficits remained. Interestingly, similar levels of exposure to the parents resulted in no findings, leading the authors to conclude that the children were more sensitive to the exposure and the deficits were most likely caused by demyelination in the 
peripheral nervous system, since mercury is known to cause demyelination in the nervous system. Kim Deitch [3], notes, “...children are almost always highly sensitive to environmental contaminants, frequently expressing signs and symptoms of toxicity at doses that fail to produce any outward indications of neurological disease in adults" (p.211).

\section{Assessment: principles and approaches}

In 2001, the World Health Organization (WHO) developed the Neurotoxicity Risk Assessment for Human Health, [4], a report that contained the collective views of an international group of experts on how to assess individuals exposed to toxic chemicals. The report indicates that, "the assessment of potential neurotoxicity in individuals begins with an individual patient in order to establish a differential diagnosis of neurotoxic disease and to rule-out other etiologies." [4]. The evaluation includes a detailed medical history and examination, and depending on the symptoms and type of exposure, may require other assessment procedures such as neuropsychological evaluation.

Neuropsychological testing has long been used as a method of documenting neurological insult as would be noted in toxic exposure, with numerous researchers and authors describing the methodology and areas that should be assessed $[5,6]$. The WHO report describes the general domains to be examined, including general intellect, attention, executive function, memory, verbal ability and language, visuospatial and visuo-motor ability, as well as mood and personality. Guidelines for conducting epidemiological studies that are more cost effective and allow for the examination of a larger number of individuals have also been published, however, these approaches often use screening measures that are less sensitive and may under-appreciate pathology or miss sub-clinical syndromes. The advantages of a neuropsychological neurotoxicology assessment are outlined by Hartman [5]. These include: 1) concurrent and predictive validity, 2) safe and non-invasive with no risk to the patient, 3) comprehensive and flexible and can assess a wide variety of cortical and subcortical functions, 4) tests are objective and replicable, 5) relatively inexpensive compared to medical and neurological workups, 6) testing is portable and can fit in one or two suitcases, 7) information is usually complimentary to conventional medical screens, and 8) because of its sensitivity the neuropsychological method can provide early warnings of potential brain damage.

\subsection{Approach to neuropsychological testing of rural Peruvian population}

For this project, the examiners reviewed the available literature in English and Spanish and consulted with other professionals on the availability of appropriate norms and instruments that were developed for specific use with a Peruvian population. Unfortunately, available measures were primarily used with the urban dwelling Peruvian individuals. Even within Peru, psychological testing of rural people such as those living in the Andes is rare. It is also remarkable that available measures commonly used by the local professionals were tests 
developed in the United States (WISC-R [7], WPPSI [8]), translated into Spanish but not re-normed on the Peruvian population. Given the questionable fairness of the test and availability of newer technologies and tests, the examiners developed adult and child protocols that were modelled after the WHO multicenter longitudinal study that explored the nature and prevalence of HIV associated neurological disorders in persons living in various geographic and social contexts [9]. This line of research developed measures that were more culture fair and with sensitivity that holds across diverse cultures and consistent with the International Test Commissions Guidelines for Test Use [10].

Demographic trends have shown Hispanics to be a growing segment of the population in the United States, driving efforts to create neuropsychological measures that can be used with Spanish speakers from various countries, taking into account education, age, and cultural variables [11-14]. Of these measures examiners opted to use the NeSBHIS [11] and the Bateria III [13], as well as other tests of motor functioning for examination of adults. For the children, WISC-IV [13] and Bateria-III, along with other measures listed below were chosen.

\subsubsection{Teacher interviews}

The protocol was piloted in 2005 and teachers within the local affected schools were interviewed at various grade levels to further examine the appropriateness of the battery and review other possible sources of information. In their system, teachers start with a set of students in the first grade and follow them through the sixth grade, such that the teacher would have the same students for six consecutive years before returning to teach a new group of first graders. All the teachers had extensive teaching experience and were present during the exposure. Three teachers were interviewed: Mrs. P ( $1^{\text {st }}$ grade teacher $), \mathrm{Mr}$. Q $\left(3^{\text {rd }}\right.$ grade teacher), and Mrs. R ( $4^{\text {th }}$ grade teacher).

3.1.1.1 Teacher interviews \#1 Mrs. P reported a sudden change in behaviour and performance among her students in the days following the mercury spill. On the day of the spill and for several days following many of the children brought mercury into the classroom or routinely played with it. From her recall, all the children in her class had direct contact with the mercury.

Mrs. P described having numerous students with visible rashes, complaints of headaches, and notable changes in their behaviour and attention. The children became very inattentive and had difficulty concentrating. They became more emotional and frequently cried or became irritable. Most of the children developed poor appetites and appeared depressed. Academically, they started having difficulty retaining information regardless of what approach to teaching was employed or how much their studying time was increased. Many of them were also noted to have difficulty with language during this period.

Mrs. P explained that where the spill occurred in June, the academic year begins in April and ends in December. In the year of the mercury spill, only 20 of her 37 students completed 1st grade requirements; over $45 \%$ of the class had to repeat the 1st grade, whereas in a normal year she typically holds back 
approximately $10 \%$ of her students. She reported that this phenomenon was not isolated to her classroom, but that the failure rate school-wide also jumped that year with approximately $30 \%$ of the school's students not graduating to the next grade.

3.1.1.2 Teacher interviews \#2 Mr. Q reported that following the mercury spill the children started to develop numerous physical problems, including rashes, headaches, vomiting, general weakness, and fatigue. Children began to miss more days of school than usual and academically appeared to become very lazy. He noted problems related to poor attention and memory, which appeared to affect their abilities in all academic pursuits. A general drop was noted in their language skills, general communication, and ability to do arithmetic. Out of a class of 26 students, Mr. Q failed eight. Mr. Q also noted that in the years to follow many of the children's problems continued. They did not seem able to recover from the exposure. In many cases, the children were pulled out of school by their parents because of their inability to perform or at the children's request that they be taken out of school. He said that at the time of the spill 350 students were enrolled at his school, but within a few years, enrollment dropped to 227 .

3.1.1.3 Teacher Interviews \#3 Mrs. R reported that in the year of the mercury spill she failed nine of her 22 students (over 40\%). She reported that many of the children described numerous physical complaints in the time immediately following the mercury exposure, including rashes, headaches, weakness, and fatigue. As the children had already developed many academic abilities and skills, they were initially able to continue or maintain what they had already learned. Academically, problems were noted starting in October and November as they got further into the academic year and were challenged with new material and development of novel skills. All the students demonstrated lower achievement and difficulty progressing.

\section{Case presentations}

Two cases are presented: Andres and Anita. They were evaluated using the following neuropsychological assessment protocol: Clinical Interview, Child Neuropsychological History Questionnaire (a structured clinical interview), Wechsler Intelligence Scales of Intelligence-IV Spanish (WISC-IV) (selected subtest of intellectual and cognitive ability), Bateria III (selected subtests of a test of achievement and aptitude), Rey Complex Figure Test (visuoperceptual and visual memory test requiring copying a complex drawing, then recalling the drawing from memory at later points), Children's Color Trails Test 1 and 2 (tests of simple sustained attention, ability to shift set, and divided attention), BeeryBuktencia Developmental Test of Visual-Motor Integration, 5th Edition - Short Form (VMI) (a measure of visual perception and graphomotor construction), Beck Youth Inventory-II (self-report measure of depression and anxiety), Grip Strength (test of gross motor hand strength), Grooved Pegboard (test of complex fine motor ability), and Finger Tapping Test (test of repetitive fine motor speed). 
The preceding measures were chosen to reduce the effects of cultural and language factors, and were given by a bilingual (Spanish/English) and bicultural (Mexican-American) examiner in the presence of a Peruvian neurologist.

\subsection{Andres's exposure}

On June 2, 2000 Andres was 2 years and 8 months old when he came in contact with elemental mercury while playing in the street near his home and later within his home. His mother reported that she and the family collected approximately 15 kilos of mercury from the roads near their home using spoons and cartons, and believing the mercury to be valuable, they brought it home. In the home they placed it on a table to separate the dirt from the mercury. Andres's mother explained that there was a lot of dirt mixed in with the mercury and that she (with the help of 14 family members) sifted out the dirt. Andres was hospitalized in June of 2000 for the effects of mercury exposure, during which time he received chelation therapy. A toxicological blood analysis done on June 12, 2000 showed a mercury level of $69.94 \mathrm{ug} / \mathrm{dl}$. Prior to the exposure, he was described as a verbal, outgoing, energetic, animated and engaging child who talked about wanting to become an attorney. After the exposure, his mother described him as having significant problems and difficulties, that included changes in his behaviour, personality, increased irritability, and being withdrawn.

\subsubsection{Andres's neuropsychological evaluation}

Andres's developmental and family history was unremarkable. He was born full term in a normal delivery, had reached developmental milestones at expected age levels, and was described as an academically average student. No systematic diseases, neurological disorders, psychiatric problems, or learning disorders were reported for Andres or his immediate and extended family.

At time of evaluation, Andres was 7 years 11 months. Andres' mother described him as having recurrent stomach problems, pain in his joints (especially his knees), and difficulties in school. In a structured interview Andres' reported problems were separated into the following three categories: 1) cognitive, 2) physical, and 3) emotional. Cognitive problems included: difficulty with novel problem solving, following directions, switching tasks, problems with decision-making, learning new tasks, poor concentration and distractibility, word-finding difficulty, poor articulation ("more like a 5 year-old"), memory problems, forgetting where he puts things, poor writing and fine motor control, easily frustrated, hypersensitive to light and sound, and very fidgety. Physical problems included: difficulty with daily headaches, urinating in his pants and bed on a daily basis, tremors, difficulty with balance, skin rashes, bone pain, and dizzy spells. Emotional problems included: sadness, poor appetite, anxiety, nervousness, difficulty falling asleep, fearful, shy and withdrawn, overly emotional, crying easily, and easily losing his temper. He is currently failing in school and has asked his mother to drop out of school.

On testing, refer to table 1, Andres appears to be in the average range of intellectual functioning. Significant difficulty in the area of nonverbal reasoning was noted, with performance in this domain being variable and ranging from 
borderline to average. On a visual memory task he demonstrated impaired recall and low average recognition, indicative of a retrieval deficit. Andres demonstrated variable mental control, with impairments in passive and divided attention. Language skills were depressed, with verbal fluency (i.e., rapid access to verbal information) in the impaired range, well below what would be expected. Verbal recall at the word and paragraph level, visuospatial and visuoconstructional abilities, and fine motor abilities were intact. His psychological profile evidenced symptoms related to depression.

\subsection{Anita's exposure}

At the time of the mercury spill Anita was 1 year and 1 month old. She was exposed to mercury while playing in the street near her home, within her home, and through nursing. Mrs. M., Anita's mother, reported that she and her family collected mercury from the roads near their home. Anita and her mother played with the mercury, put it on their bodies and stored the mercury in their home. When the mining company became aware of the mercury spill the company set up locations to collect the mercury and reward the individuals who returned it. When Mrs. M. attempted to return the mercury she was told that it had too much dirt and to return after she had cleaned it, and was instructed to boil the dirt out. Mrs. M. boiled the mercury over an open wood fire in her home where ventilation was limited; the walls of her home are constructed of the local dirt with dirt floors. Anita developed a rash, problems breathing, head aches, change in personality (irritability), problems sleeping and had blood in her feces. She was hospitalized in June of 2000 for the effects of mercury exposure and received chelation therapy and was classified as "red code" with risk of organ damage because of her young age. Her body was still developing and was in a critical period. In February 2002 she showed low creatinine depuration denoting renal compromise. In August 2004 Anita continued having breathing problems with wheezing, chronic headaches and chronic dermatitis. Notes by local medical providers noted her to have normal intelligence but with deficits in visual organization and concentration.

\subsubsection{Anita's neuropsychological evaluation}

Anita's developmental, medical and family history was unremarkable. At time of evaluation she was 6 years old. Her teacher described her as having significant difficulty in reading, writing and academically falling further behind than her peers and expected that over time the gap would widen. She took 3 to 4 hours per day to complete her homework and required constant supervision and tutoring. Anita was notably reserved, less social than her peers, and presented as depressed. She was quick to react and was often aggressive.

On interview, Mrs. M reported that Anita was very active and distractible. In the period following the exposure Anita was described as very withdrawn and difficult to sooth, but over the years received extensive intervention and treatment, through schools, tutors, doctors, and therapists. A level of care and attention that is not typical for most of the individuals exposed. Cognitive problems endorsed: difficulties with novel problem solving, following directions, 


\section{Management of Natural Resources, Sustainable Development and Ecological Hazards}

switching tasks, learning new tasks, poor concentration and distractibility, easily losing train of thought, and memory problems. Physical symptoms endorsed: occasional headaches, urinates in her pants and bed, skin rashes, bone pain (in her joints which often makes her cry), and very fidgety with difficulty standing still. Emotional problems reported: poor appetite, difficulty with sleep initiation, periodic nightmares, depression, anxiety, nervousness, shy and withdrawn, emotional, crying easily, easily loses temper, aggressive and easily frustrated.

Table 1: $\quad$ Interpretation of neuropsychological testing.

\begin{tabular}{|c|c|c|}
\hline INTELLECTUAL & Anita & Andres \\
\hline Raven's Colored Progressive Matrices & Average & Average \\
\hline Digit Span-WISC-IV & Impaired & High Average \\
\hline Coding-WISC-IV & Borderline & Average \\
\hline Block Design -WISC-IV & Average & High Average \\
\hline Matrix Reasoning-WISC-IV & Average & Impaired \\
\hline Symbol Search-WISC-IV & Average & Low Average \\
\hline Processing Speed Index & Low Average & Average \\
\hline \multicolumn{3}{|l|}{ LANGUAGE } \\
\hline Picture Vocabulary-Bateria-III & Low Average & Impaired \\
\hline Verbal Fluency-Bateria-III & Average & Average \\
\hline Oral Expression-Bateria III & Low average & Low Average \\
\hline \multicolumn{3}{|l|}{ MENTAL CONTROL } \\
\hline Color Trails 1 & Impaired & Borderline \\
\hline Color Trails 2 & Impaired & Average \\
\hline Digit Span-WISC-IV & Impaired & Average \\
\hline Coding-WISC-IV & Borderline & Borderline \\
\hline \multicolumn{3}{|l|}{ VISUOSPATIAL } \\
\hline Raven's Progressive Matrices & Average & Average \\
\hline Rey Complex Figure Copy & Discontinued & Average \\
\hline Block Design-WISC-IV & Average & High Average \\
\hline Bender-Gestalt (Error) & Impaired & Intact \\
\hline \multicolumn{3}{|l|}{ MEMORY-VISUAL } \\
\hline Rey Complex Figure Immediate & Discontinued & Impaired \\
\hline Rey Complex Figure Delayed & Discontinued & Impaired \\
\hline Rey Complex Figure Recognition & Discontinued & Low Average \\
\hline Design Recall-Bateria-III & Low Average & Average \\
\hline \multicolumn{3}{|l|}{ MEMORY-VERBAL } \\
\hline Story Recall -Bateria-III (3) & Low Average & Impaired \\
\hline Story Recall (Delayed)-Bateria-III (12) & Impaired & Impaired \\
\hline Word Memory-Bateria III & Average & Low average \\
\hline
\end{tabular}


On testing, Anita presented with average intellectual ability. Language skills were in the low average to average range. Mental control was generally impaired and reflecting difficulty with attention. She did well on non-motor visuospatial tasks. When tasks required the integration of fine motor and visual spatial abilities she demonstrated severe deficits, not being able to complete one task. On a similar task not requiring fine motor ability, she performed in the low average range. Immediate short-term verbal memory was grossly intact, while delayed verbal memory was impaired.

\section{Discussion of neuropsychological test results}

Testing provided meaningful data, with the selected tests demonstrating appropriateness in terms of language (appropriate Spanish) and findings consistent with the literature and collateral information. In reviewing Andres's and Anita's performance, it is interesting to note the scatter or variability in scores within the various domains assessed (mental control, intelligence, language, visual spatial and memory). Their scores indicate potential for average to high average performance in all domains; however, on specific tests measuring different aspects of those same domains, performance is impaired. Examination of the commonalities suggest possible executive dysfunction, with what appears to be planning, organizational and monitoring difficulty, as well as deficits or processing problems in the area of information retrieval, all of which would significantly impair their ability to function, especially in school. It is well within the realm of medical probability that current difficulties reflect the effect of mercury on Andres' neurodevelopment, given that his exposure to mercury occurred at a time of rapid neurodevelopment. The noted difficulties are consistent with collateral information and reflective of deficits that will have real world implications. They suggest children having difficulty functioning within their school and home environments, despite, in Anita's case, a heroic level of intervention. Given the time elapsed since their exposure and the limited and questionable improvement it is highly likely that both will have persistent impairments and disabilities for life. Will they continue to fall behind, maintain or improve? Only further research can answer that question. At a societal level, the implications of the mercury spill are immeasurable, especially if one considers the number of children affected, the rate of school drop out, and the loss of intellectual ability in a community with limited resources for remediation. These children are growing up with valid psychological fears for their future and well being.

\section{References}

[1] ATSDR (1999) Toxicological profile for mercury (update). Atlanta, GA, US Department of Health and Human Services, Public Health Services, Agency for Toxic Substances and Disease registry, March. 
[2] Rohling ML \& Demakis GJ (2006) A meta-analysis of the neuropsychological effects of occupational exposure to mercury. The Clinical Neuropsychologist, 20, pp 108-132.

[3] Deitch K (2000) Environmental toxicants and psychological development. In Yeates, Ris \& Taylor eds. Pediatric Neuropsychology: Research, Theory, and Practice. Guilford Press, New York

[4] WHO (2001) Environmental Health Criteria 223 Neurotoxicity Risk Assessment for Human Health: Principles and approaches, Geneva.

[5] Hartman DE (1995) Neuropsychological toxicology: Identification and assessment of human neurotoxic syndromes, $1^{\text {st }}$ ed. New York, Pergamon Press.

[6] White RF \& Proctor Sp (1995) Clinico-neuropsychological assessment methods in behavioural Neurotoxicology. In: Chang LW \& Slikker W. ed Neurotoxicology: Approaches and methods. New York, Academic Press, pp 711-726.

[7] Weschler D (1976) Weschler Intelligence Scale for Children-Revised. New York: Psychological Corporation.

[8] Wechsler D (1967) Wechsler Preschool and Primary Scale of Intelligence -Revised. New York: Psychological Corporation.

[9] Maj M, D'Elia, L, Jannsen R, Zaudig, M, Uchiyama, C, Starace F, Galdererisi S \& Chervinsky, A (1993) Evaluation of three new neuropsychological test designed to minimize cultural bias in the assessment of HIV-1 Seropositive persons: A WHO Study. Archives of Clinical Neuropsychology, 8 123-135.

[10] International Test Commission (2000) International Guidelines for Test Use, Stockholm.

[11] Ponton MO, Satz P, Herrera L, Ortiz, F, Furst, C \& Namerow, N (1996) Normative Data stratified by age and education for the neuropsychological screening battery for Hispanics (NeSBHIS): Initial report. Journal of the International Neuropsychological Society 2 (2), 96104.

[12] Artiola IF, Hermosilla D, Heaton RK \& Pardee RE (1999) Manual de normas y procedimientos para la Bateria Neuropsicologic en Espanol, Tucson, $\mathrm{m}$ Press.

[13] Woodcock RW, Muñoz-Sandoval AM, McGrew, KS \& Nancy M (2005) Bateria III Woodcock-Munoz, Riverside Publishing.

[14] Wechsler D (2004) Wechsler Intelligence Scale for Children-IV Spanish. San Antonio: Psychological Corporation. 